\title{
Activation of Neurons in Cardiovascular Areas of Cat Brain Stem Affects Spinal Reflexes
}

\author{
W. C. WU, ${ }^{*} \dagger$ S. D. WANG, $\ddagger$ J. C. LIU, $\ddagger$ H. T. HORNG, ${ }^{*}$ M. J. WAYNER, $\S$ \\ J. C. MA† AND C. Y. CHAI*1 \\ *Institute of Biomedical Sciences, Academia Sinica, Nankang 11529, Taipei, †Department of Electrical Engineering, \\ National Taiwan University, $\ddagger$ Department of Biology and Anatomy, National Defense Medical Center, Taipei, \\ Taiwan, ROC, \$Division of Life Sciences, University of Texas at San Antonio, San Antonio, TX 78285
}

Received 1 February 1993; Accepted 18 August 1993

\begin{abstract}
WU, W. C., S. D. WANG, J. C. LU, H. T. HORNG, M. J. WAYNER, J. C. MA AND C. Y. CHAI. Activation of neurons in cardiovascular areas of cat brain stem affects spinal reflexes. BRAIN RES BULL 33(4) 393-402, 1994.-In 65 cats anesthetized with chloralose $(40 \mathrm{mg} / \mathrm{kg}$ ) and urethane $(400 \mathrm{mg} / \mathrm{kg}$ ), the effects of electrical stimulation and microinjection of sodium glutamate $(0.25 \mathrm{M}, 100-200 \mathrm{nl})$ in the pressor areas in the rostral brain stem on the evoked LS ventral root response (EVRR) due to intermittent stimulation of sciatic afferents were compared to stimulating the dorsomedial (DM) and ventrolateral (VLM) medulla. In general, stimulating these rostral brain stem pressor areas including the diencephalon (DIC) and rostral pons (RP) produced increases in systemic arterial pressure (SAP). In most of the cases (85\%) there were associated changes in the EVRR, predominantly a decrease in EVRR (72\%). Stimulation of the midbrain (MB, principally in the periaqueductal grey) produced decreases in SAP and EVRR. Decreases in EVRR was observed in $91 \%$ of the DM and VLM stimulations in which an increase in SAP was produced. This EVRR inhibition was essentially unaltered after acute midcollicular decerebration. Increases in EVRR were also observed and occurred more often in the rostral brain stem than in the medulla. Since changes of both EVRR and SAP could be reproduced by microinjection of Glu into the cardiovascular-reactive areas of the brain stem, this suggests that neuronal perikarya in these areas are responsible for both actions. On some occasions, Glu induced changes in EVRR but not in SAP. This effect occurred more frequently in the rostral brain stem than in the medulla. The present data suggest that separate neuron population exist in the brain stem for the integration of SAP and spinal reflexes. These neurons are relatively dispersed from DIC to RP but are in close proximity to one another in the medulla. Rostral structures also contain more neurons responsible for augmenting spinal reflexes.
\end{abstract}

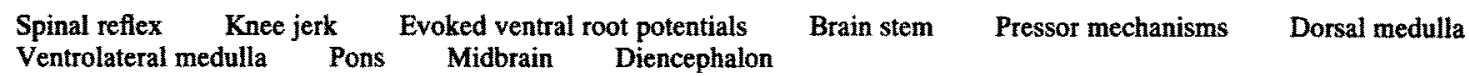

OUR previous studies have shown that two separate areas of the dorsomedial (DM) and ventrolateral (VLM) medulla contain perikarya which can increase systemic arterial pressure (SAP) in various species $(6,23,24,32)$. It is also known that many neural mechanisms important for cardiovascular integration exist in the rostral brain stem as well as in the medulla oblongata. These major areas are the locus coeruleus complex (LC) of the rostral pons, periaqueductal grey of the midbrain $(1,21,22)$, and the hypothalamus of the diencephalon. We and other investigators have also found other pressor response areas in the dorsolateral region of the pons $(8,21)$.

Recently, we demonstrated the co-existence of neuronal subpopulations in the sympathetic pressor areas of the DM, VLM and parvocellular nucleus (PVC) of medulla oblongata (8) that integrate spinal reflexes, that is, knce jerk (KnJ), crossed extension movements (CEM) and evoked L5 ventral root response (EVRR), induced by stimulation of the central end of the sciatic or tibial nerve. During stimulation of these pressor areas, marked inhibition of spinal reflexes and increases in SAP was observed. This inhibition was found to be independent of potential recip- rocal connections with the cerebellum (7) and the powerful inhibitory mechanism in the paramedian reticular nucleus of the caudal medulla (8). These results suggest a coexistence of neuronal populations in the DM, VLM, and PVC that affect both the sympathetic pressor systems and somatic spinal reflex.

The purpose of the present study was to extend our previous experiments of EVRR in response to medulla oblongata stimulation to stimulation of the pressor areas of the more rostral structures in the rostral pons (RP), midbrain (MB), and diencephalon (DIC) of the brain stem. It was also to determine if these pressor areas in the rostral brain stem contain perikarya which affect both SAP and EVRR. Microinjection of Glu was used because it only activates the dendrites and cell bodies of neurons but not axons and thus spares all fibers of passage (15).

\section{MATERIALS AND METHODS}

Sixty five cats of either sex, weighting $2.0-3.6 \mathrm{~kg}$, anesthetized with a mixture of urethane $(400 \mathrm{mg} / \mathrm{kg}$ ) and alpha chloralose $(40 \mathrm{mg} / \mathrm{kg})$ administered intraperitoneally were used. The

\footnotetext{
${ }^{1}$ To whom requests for reprints should be addressed.
} 
general procedures for experimentation have been described previously (4-8). These included cannulation of the right femoral artery for monitoring the systcmic blood pressure (SAP) and the mean SAP (MSAP) which triggered the heart rate (HR), cannulation of the right femoral vein for drugs injections, tracheal intubation for artificial ventilation maintaining the end-expiratory $\mathrm{CO}_{2}$ concentration at $4 \%$, and keeping rectal temperature at $37.5^{\circ} \mathrm{C}$ by a thermostatically controlled heating pad. All recordings were made on a Gould ES1000 recorder. The responses were also taped by a HP $3968 \mathrm{~A}$ recorder for later analysis.

\section{Spinal Reflexes}

Production of spinal reflexes, the evoked ventral root response (EVRR) following activation of the spinal afferents, has been described previously (4-8). In brief, the animal was put on a David-Kopf spinal investigation unit. The animal was further paralyzed by atracurium besylate $(0.5 \mathrm{mg} / \mathrm{kg} / 30 \mathrm{~min}) \mathrm{IV}$. The spinal cord from L4 to L6 was exposed and together with the nerves were immersed in paraffin oil. The ventral rootlets at L5 were isolated and cut. Its central end was hooked on a bipolar platinum electrode for recording the evoked potentials induced every second by stimulation of the ipsilateral tibial nerve with rectangular pulses $(1 \mathrm{msec}, 1 \mathrm{~Hz}$ and $200-500 \mu \mathrm{A})$ from a Grass S-88 stimulator coupled to a constant current unit. The signals were amplified and filtered with a WPI preamplifier (DAM 60 , $100-1000 X, 1 \mathrm{~Hz}$ to $3 \mathrm{kHz}$ ) and a Gould universal amplifier. Signals were displayed on a storage oscilloscope (Tektronix 5113). For analysis of the EVRR, the evoked potential was expanded and displayed on the polygraph through a waveform module (Gould, $100 \mathrm{kHz}$ ). Four components of the EVRR were observed: the short latency $(2.5 \mathrm{~ms})$ component 1 and the long latency component $2(4.5 \mathrm{~ms}), 3(9.0 \mathrm{~ms})$, and $4(20.0 \mathrm{~ms})$. Only components 1 and 2 were retained and amplified for illustrations and calculation. The amplitude of component 2 was used as an index of the intensity of spinal reflexes.

\section{Brain Stimulation}

Brain stimulation, either electrically (monopolor, $80 \mathrm{~Hz}, 1$ msec, $100-400 \mu \mathrm{A}$ for $15 \mathrm{~s}$ from a Grass S-88 stimulator) or chemically, was accomplished through the same electrode-array which was made of $30 \mathrm{G}$ needle tubing connected to a $10 \mu \mathrm{l}$ Hamillton syringe. The electrode was filled with monosodium glutamate (Glu, 0.25-0.5 M, pH 7.4) in artificial cerebrospinal fluid containing fast green as a marker (8). The electrode-array was inserted into the RP, DM, or VLM at an angle of $34^{\circ}$ while in DIC or $\mathrm{MB}$ at rectangle. For making brain lesions direct current $(D C, 1 \mathrm{~mA})$ for $50 \mathrm{~s}$ was passed through the electrode tubing.

\section{Decerebration}

Decerebration was performed at the mid-collicular level with ligation of both external carotid arteries and clamping of the basilar artery as has been described previously $(7,8)$.

\section{Histology}

At the end of each experiment, the animal was sacrificed with an overdose of pentobarbital. The fresh brain was removed and immersed in saturated formalin for $2 \mathrm{~min}$; the chemical injection sites were verified using two consecutive frozen sections, at $50 \mu \mathrm{m}$ thick one stained with cresyl violet for identifying nuclei, and the other unstained for determing the position and diffusion of dye.

\section{Data Analysis}

In the experiments on brain stimulation the significance of the percent changes of SAP and EVRR from the control were determined by Student's $t$-test. Statistical significance was set at $p<0.05$. The effectiveness of each response from its control level was tested against a hypothesis that the percent change before the stimulation was zero.

In experiments on brain lesioning, effects before and after lesions were compared in the same animals by paired Student's $t$-test. A one-way analysis of variance (ANOVA) was performed for experimental group comparisons and statistical significance for specific comparisons was set at $p<0.05$.

\section{RESULTS}

\section{Stimulation Sites in the Cardiovascular Areas of the Brain Stem That Produced Changes in the Activity Pattern of Spinal Reflexes}

Sites. The cardiovascular reactive sites in the rostral brain stem and medulla oblongata were explored first by electrical stimulation with rectangular pulses and then confirmed by Glu microinjection for neuronal cell body activation. All together, 73 in DIC, 56 in MB and 109 in RP of the rostral brain stem and 37 in DM and 29 in VLM of the medulla oblongata responded to Glu in producing various changes of SAP or EVRR. These reactive sites of the rostral brain stem, located from the diencephalon through the rostral pons in different combinations, are mapped in Fig. 1. In the diencephalon region, these reactive sites were found principally in the ventromedial [28], dorsal [24], and parvocellular nuclei [16] of the hypothalamus; and a few points [5] were located in the anterior nucleus of the thalamus. In the midbrain region, most the reactive sites were found in the periaqueductal grey [PAG, 38] and the central tegmental field [18]. In the rostral pons region, sites were distributed in the locus coeruleus [LC, 20], gigantocellular tegmental field [FTG, 45], and lateral tegmental field [FTL, 44]. The locations of reactive sites in the DM [37] and VLM [29] in the medulla oblongata are not included in Fig. 1 because they were the same as reported previously (6).

Table 1 summarizes the responses of these reactive sites in three groups based on the changes of SAP, that is, increase, decrease, or no change following Glu microinjection. Also three types of changes in EVRR; that is, increase, decrease, or no change are included.

Pattern. The onset and duration of the induced responses following electrical or Glu stimulation were the same in both the rostral brain stem and the medulla oblongata (8). Nevertheless, differences were observed between electrical and Glu activation. Changes of EVRR were not always produced simultaneously with changes of SAP due to brain stimulation. In medulla oblongata, when Glu produced changes of EVRR, it was always concomitant with an increase in SAP. In such cases, electrical stimulation also produced the same changes of EVRR and SAP as Glu. In DIC and RP, Glu might induce changes in EVRR not concomitant with changes in SAP (58/68 and 68/102, respectively), while electrical stimulation produced changes in SAP concomitant (Fig. 2)(68/73 and 102/109, respectively) or not concomitant (5/73 and 7/109) with changes in EVRR. In MB, 34 loci in PAG and 7 loci in its adjacent areas, responded to electrical stimulation, but not Glu, by increases in SAP (average of $68 \mathrm{mmHg}$ ). Nevertheless, they responded to Glu in producing changes in EVRR (Fig. 3). Among them, EVRR was decreased $(39.5 \%)$ by stimulation of the PAG while increases $(11.1 \%)$ were observed when the adjacent areas of the PAG were stimulated. 


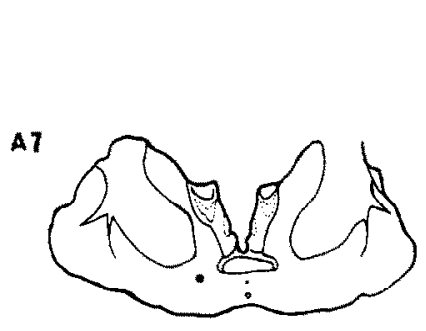

A4

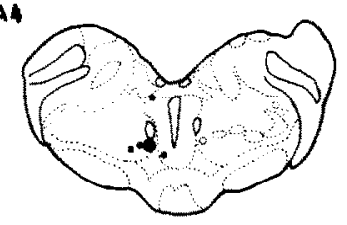

A3
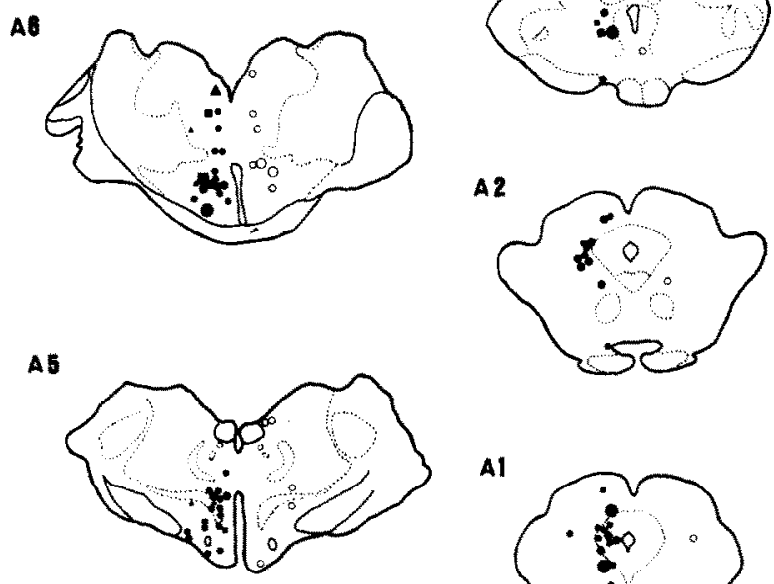

A1
0

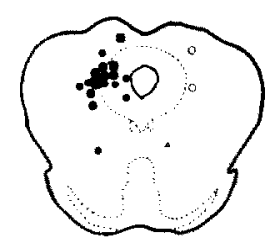

P1

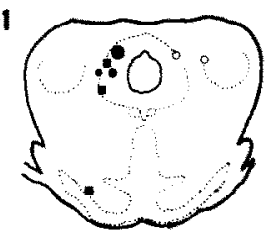

P2

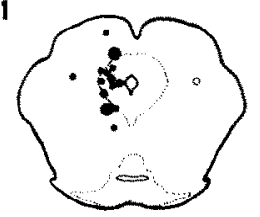

$5 \mathbf{m m}$

012

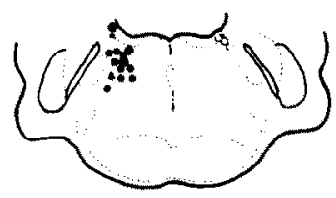

OII

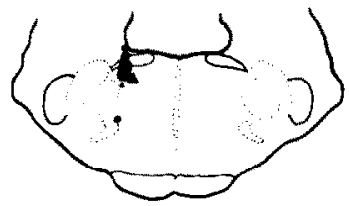

010

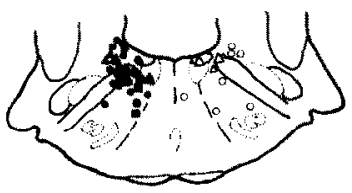

og

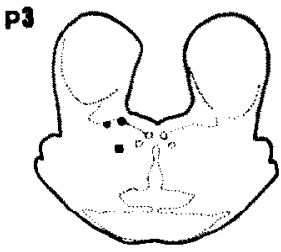

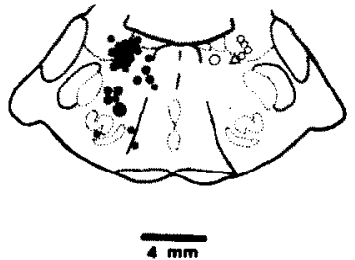

FIG. 1. Distribution of reactive sites affecting spinal reflexes in the sympathetic pressor areas of the brain stem. Stimulation in diencephalon-midbrain was carried out from the level of $7 \mathrm{~mm}$ anterior (A7), to $3 \mathrm{~mm}$ (P3) posterior to the ear bar zero with the electrode-tubing setting at right angle, while ponto-medulla was from the level of $9 \mathrm{~mm}(09)$ to $12 \mathrm{~mm}$ rostral to the obex with the electrode-tubing setting at $34^{\circ}$. Fig. 1 shows brain drawings from $A 7$ to $A 1$, zero-P3, O12-O9. Sites that caused inhibition of the reflexes are mapped on the left half and augmentation of the refiexes are mapped on the right half of the brain drawing. Solid circles $(\bullet)$ are used to mark sites that produced reduction of EVRR only, without associated SAP changes. Three different sizes are used to indicate the extent of reduction in decreasing order, from $60-100 \%, 30-60 \%$, and 5-30\%, respectively. Three different sizes of solid triangles $(A)$ are used to indicate reduction of EVRR associated with increases in SAP in decreasing order from $60-100 \%$, $30-60 \%$, and 5-30\%, respectively. Three different sizes of solid squares (D) are used to indicate the EVRR reduction associated with a decrease in SAP in a decreasing order from $60-100 \%, 30-60 \%$, and $5-30 \%$, respectively. Two different sizes of large and small empty circles (O) are used to indicate the increase of EVRR augmentation without associated SAP changes, large circles from $30-60 \%$ and small circles from $5-30 \%$, respectively. Three different sizes of empty triangles $(\Delta)$ are used to indicate the increases of EVRR associated with a increase in SAP in decreasing order from $60 \%$ or more, $30-60 \%$, and $5-30 \%$, respectively.

The sensitivity of changes in SAP and EVRR between DIC and MB due to electrical stimulation was tested in some cases. The EVRR component was more sensitive; it required only $100 \mu \mathrm{A}$ to produce an apparent change. The SAP component was less sensitive; it required $300 \mu \mathrm{A}$ to produce the response. In DM and VLM $100 \mu \mathrm{A}$ was sufficient to produce marked responses both in EVRR and SAP.

The reactivity of various brain structures to Glu was also compared (Table 1). Among the DIC and RP that received the same dose of Glu microinjection, the degree of MSAP increase was weakest in DIC (5.1\%), yet its effects on EVRR were apparent (decreased $25.8-34.1 \%$ or increased $16.2 \%$ ). Glu stimulation of the RP region produced the highest MSAP increase (41.2\%). Glu stimulation of the MB region principally produced no change or decrease in MSAP (9.8\%). EVRR decreased (37.2-41.4\%) or increased $(11.1 \%)$, Glu stimulation of the RP region produced no change, increase $(41.2 \%)$ or decrease $(9.2 \%)$ in MSAP. EVRR also decreased $(24.6-42.4 \%)$ or increase $(14.4-51.6 \%)$.

\section{Activation Produced Inhibition of Spinal Reflexes}

Changes in spinal reflexes, in terms of EVRR inhibition produced by chemical stimulation in the brain stem, 51 in DIC, 48 in MB, 84 in RP, 24 in VLM, and 36 in DM, are illustrated in Fig. 1 and Table. 1 . In the rostral brain stem, the major change in EVRR activity was inhibition associated with no change in SAP (41/51 in DIC, 38/48 in MB, and 56/84 in RP). These effects were observed only in a few cases following DM (6/36) and VLM (6/24) stimulation. However, when DM (21/36) or VLM $(15 / 24)$ was activated, it produced EVRR inhibition associated with an increase of SAP.

With Glu stimulation, the percentage of time when EVRR inhibition was observed simultaneously with SAP increases was 
TABLE 1

EFFECTS OF GLU BRAIN STIMULATION ON EVRR

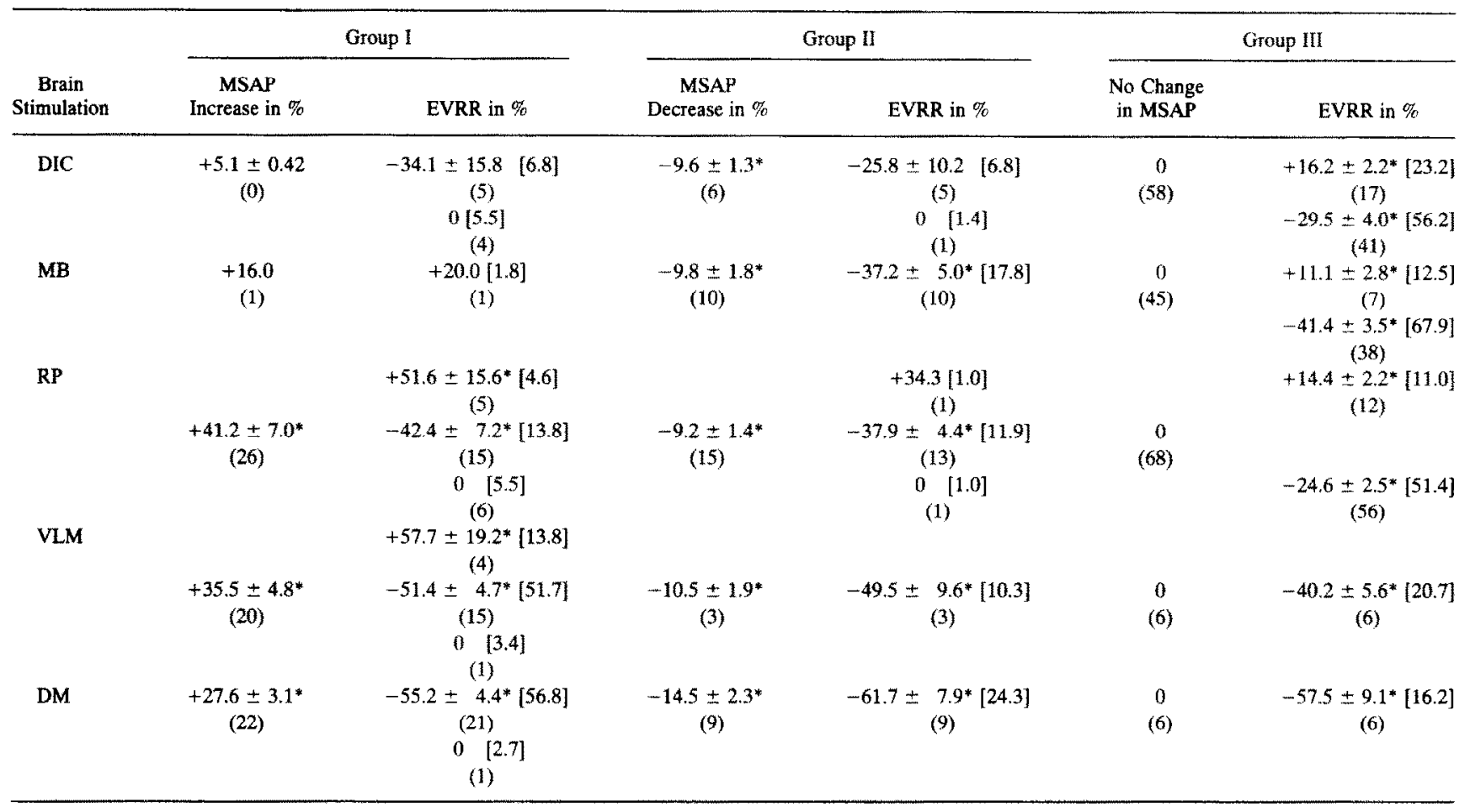

DIC: Diencephalon; MB: Midbrain; RP: Rostral pons; VLM: Ventrolateral medulla; DM: Dorsal medulla; MSAP: Mean systemic arterial blood pressure (mmHg); EVRR: Evoked ventral root response.

$+:$ Increase; -: Decrease; 0: No change.

All values are mean $\pm \mathrm{SD} ;(n)=$ number of brain sites receiving Glu microinjection; $[N]=$ frequency of observation, in $\%$, during brain stimulation.

* Values before and after glutamate (Glu) stimulation of brain are statistically significant calculated by Student's $t$-test with $p$ values $<0.05$.

as follows: DM 56.8, VLM 51.7, RP 13.8, DIC 6.8. The percentage of time EVRR inhibition was associated with SAP decreases was: DM 24.3, MB 17.8, RP 11.9, VLM 10.3, DIC 6.8. On the other hand, decreases of EVRR associated without any or only a very slight change (less than 5\%) in SAP was observed much more often in the rostral than the caudal structures. The percentage in decreasing order was: MB 67.9, DIC 56.2, RP 51.4, VLM 20.7, and DM 16.2.

In general, neurons that inhibited EVRR were found over the whole brain stem, from diencephalon to medulla without any specific localization. The only difference was that location of these neurons in the rostral structures, that is, DIC, MB, and RP, was not that close to the neurons mediating sympathetic pressor action, in comparison to the caudal structures, that is, VLM and DM.

\section{Activation Produced Excitation of Spinal Reflexes}

Increases in EVRR activity were also observed following Glu stimulation but less frequently: (a) In 11 cases; PAG ( 1 of the 46 stimulations), RP ( 6 of the 26 stimulations), and VLM (4 of the 20 stimulations) were associated with increase in SAP (Figs. 4 and 5). (b) Increased EVRR associated with a decrease of SAP was observed only once in RP. The other observations in percentage were associated with increases in SAP: VLM 13.8, RP 4.6, and MB 1.8. (c) In 36 areas (DIC 17, MB 7, RP 12) the increases in EVRR were not associated with changes in SAP. The percentages were: DIC 23.2, MB 12.5, RP 11.0.
These data show that neurons mediating an increase in EVRR were localized more in the structures in or rostral to RP than in the caudal structures of VLM and DM.

However, even though neurons which increase or decrease in EVRR were found in the same area such as RP, a comparison of Figs. 4 and 6 shows that microinjection of Glu into the gigantocellular reticular tegmental field (FTG) of RP which increased MSAP have an opposite effect on EVRR. The two injections were close together, but one produced an increase in EVRR (Fig. 4) while the other decreased EVRR (Fig. 6).

In 25 animals, SAP and EVRR due to electrical stimulation of the pressor areas in DIC, MB, or RP were measured before and after direct current lesions were made in various pressor areas caudal to the stimulating sites. Results are summarized in Table 2. Lesions in the pressor area of the VLM produced a significant reduction of the pressor response and decrease in EVRR in response to DIC and RP stimulation. However, after VLM lesions, the EVRR following MB stimulation was significantly increased despite a reduction of the pressor response. Lesioning of the PAG caused a significant reduction of EVRR due to DIC stimulation. Lesioning of the RP significantly increased the EVRR in response to MB stimulation.

\section{Activation Producing Only SAP Changes}

Chances of observing a change in SAP with or without only a small change in EVRR due to brain stimulation with Glu was small (Fig. 7); DIC (5 of 73 stimulations; 4 pressor, 1 depressor), 
$\mathbf{0 0 0 7 1 0}$

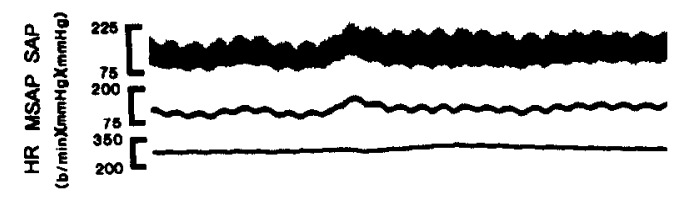

$\stackrel{\alpha}{\mathbb{\alpha}}$
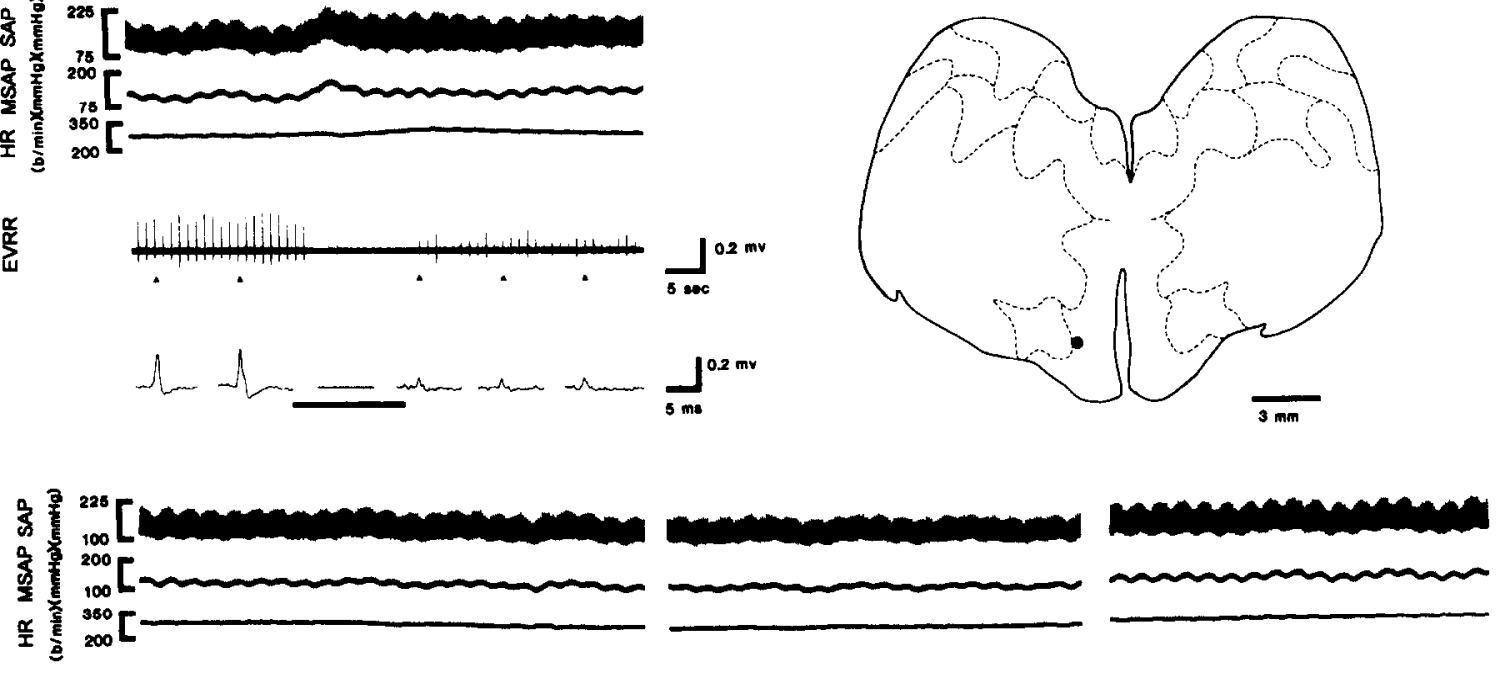

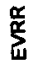
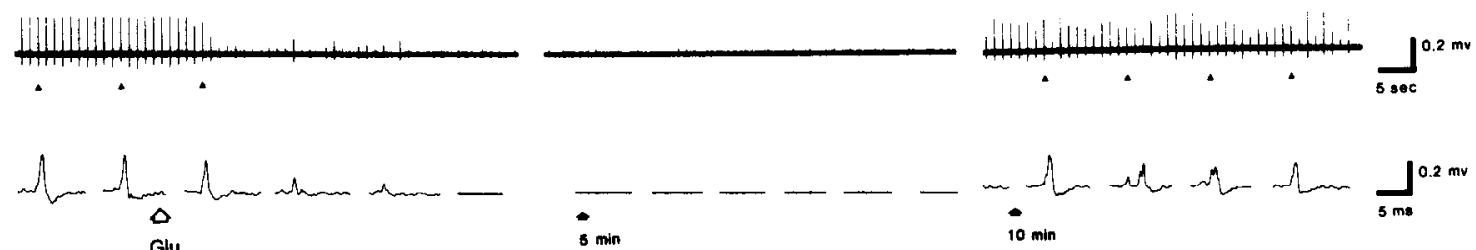

FIG. 2. Stimulation of the diencephalon (DIC) produced inhibition of EVRR. In this and the following figures, traces from top to bottom, SAP (systemic arterial blood pressure), MSAP (mean SAP), HR (heart rate) and EVRR (evoked ventral root responses) and the expanded EVRR from a specific EVRR indicated by arrowheads $(\Delta)$. Bar $(-)$ and arrow $(\Delta)$ indicate time of delivering electrical stimulation and microinjection of Glu respectively. EVRR were induced by stimulating the central end of the cut sciatic nerve (left) every $s$ with a single pulse $(100 \mu \mathrm{A}, 1.0 \mathrm{msec})$. The DIC (dot in the brain drawing) was stimulated first with an electrical current $(400 \mu \mathrm{A}, 1.0 \mathrm{msec}, 80 \mathrm{~Hz}$ and $15 \mathrm{~s}$ ) (upper panel) and then followed by Glu $(200 \mathrm{nl}$ ) (lower panel). Note that electrical stimulation produced an increase of SAP and a decrease of EVRR. Glu stimulation produced a mild decrease of SAP and complete abolition of EVRR.

RP (7 of 109 stimulations; 6 pressor, 1 depressor), VLM (1 of 29 stimulations, pressor), DM (1 of 37 stimulations, pressor).

\section{Role of the Rostral Neural Structures in Changes of EVRR Produced by RP, VLM or DM Stimulation}

In 5 cats inhibition or excitation of EVRR induced by either electrical or chemical stimulation of the RP, VLM, or DM stimulation was compared before and after acute midcollicular decerebration. Decerebration essentially had no effect on the resting SAP and EVRR induced by stimulation of the various pressor areas of the brain stem. EVRR due to stimulation of the RP, VLM, and DM was not altered by further direct current lesions in the pressor areas of MB on both sides.

\section{DISCUSSION}

Results show clearly that stimulation of DIC, MB, and RP produces changes of SAP or EVRR in various ways. The most common effect was a decrease in EVRR and an increase in SAP. A second but less common occurrence was a decrease in EVRR without any change in SAP. Only in a few cases did stimulation produce a decrease in EVRR and a decrease in SAP. These data are similar to our previous results on DM and VLM on EVRR (8). However, the medullary stimulated increase in EVRR was not observed frequently.

In our previous study (8) it was shown that the effects of EVRR following DM and VLM stimulation were independent of the cerebellum and neural structures rostral to the midbrain and were not related to the vagus nerve and the baroreceptor reflex. In both of our studies, modulation of SAP and EVRR was produced by Glu stimulation of DIC, RP, DM and VLM. This suggests that activation of perikarya instead of fibers of passage are responsible for the actions (15). The SAP response was relatively insensitive to Glu stimulation of the $\mathrm{MB}$, although in some cases it produced changes in EVRR. This suggests that changes of SAP due to MB activation probably involve principally fibers of passage, or Glu is not the appropriate chemical for activation. Both the present and previous results (8) demonstrated that decreased or increased EVRR could be produced without an associated with change in SAP, or vice versa. Furthermore, changes of EVRR required a much lower intensity of stimulating current than that of SAP. This suggests that the EVRR and SAP mechanism are independent. A similar dissociation of antinociceptive effects on SAP following microinjection of Glu in VLM has also been observed (18).

In the more rostral DIC and MB as compared to the caudal DM and VLM, stimulation induced more often changes in EVRR without any change in SAP as can be seen in Table 1 . Also increased EVRR amplitude was observed more frequently during stimulation of the rostral structures. These results are consistent with the early data of Magoun (25) who found that the motor responses (blink, flexor, and patellar reflex) produced by electrical stimulation of the motor cortex were facilitated more by diencephalon structures than in other regions of the brain stem 

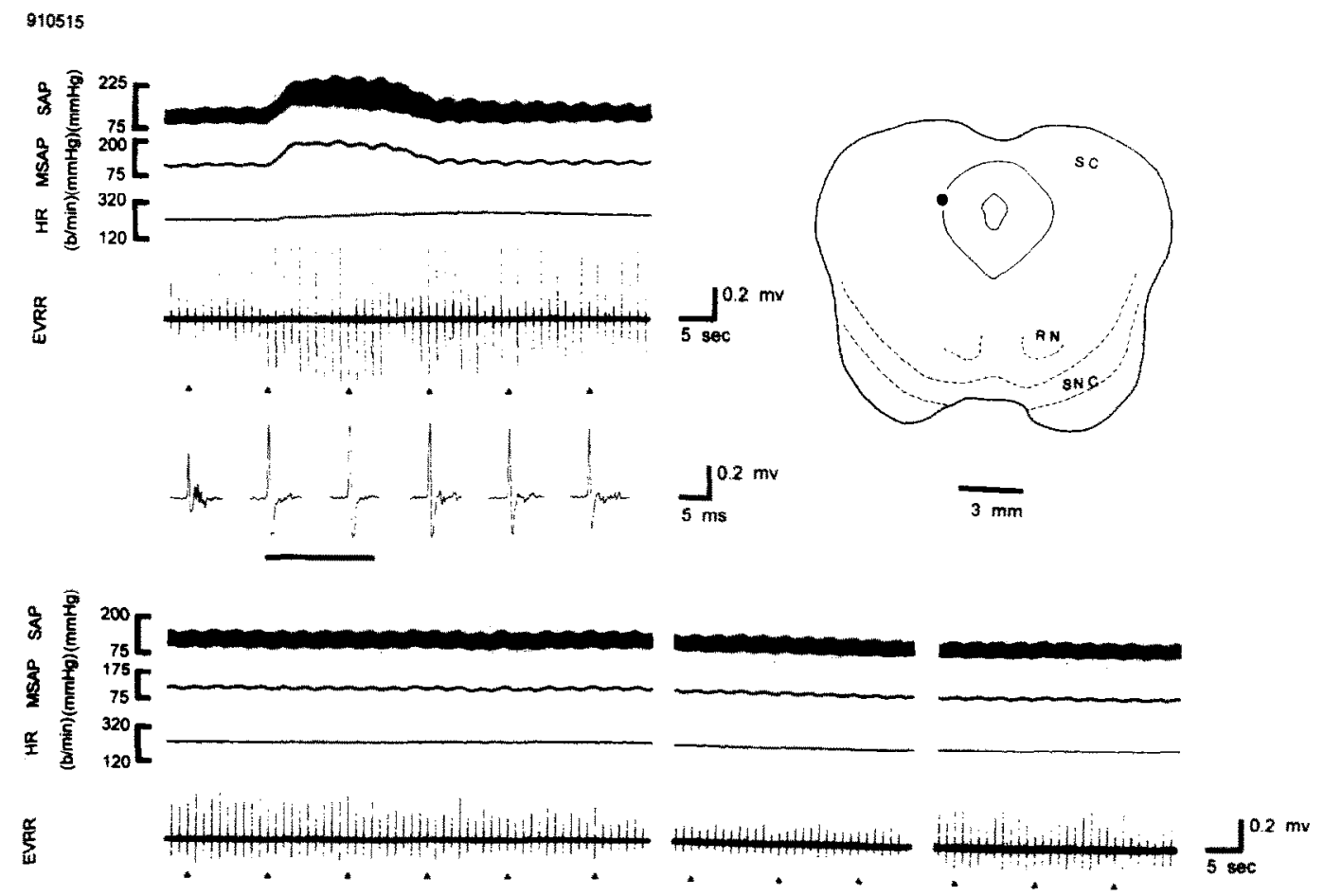

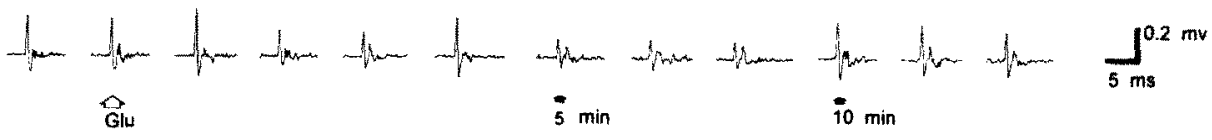
FIG. 3.

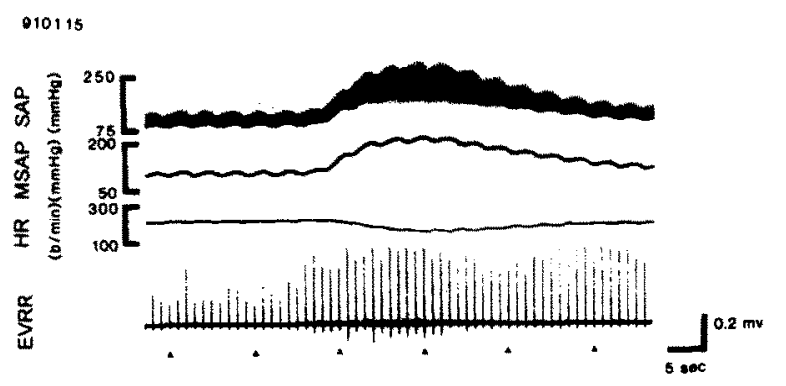

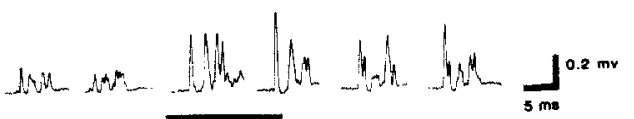
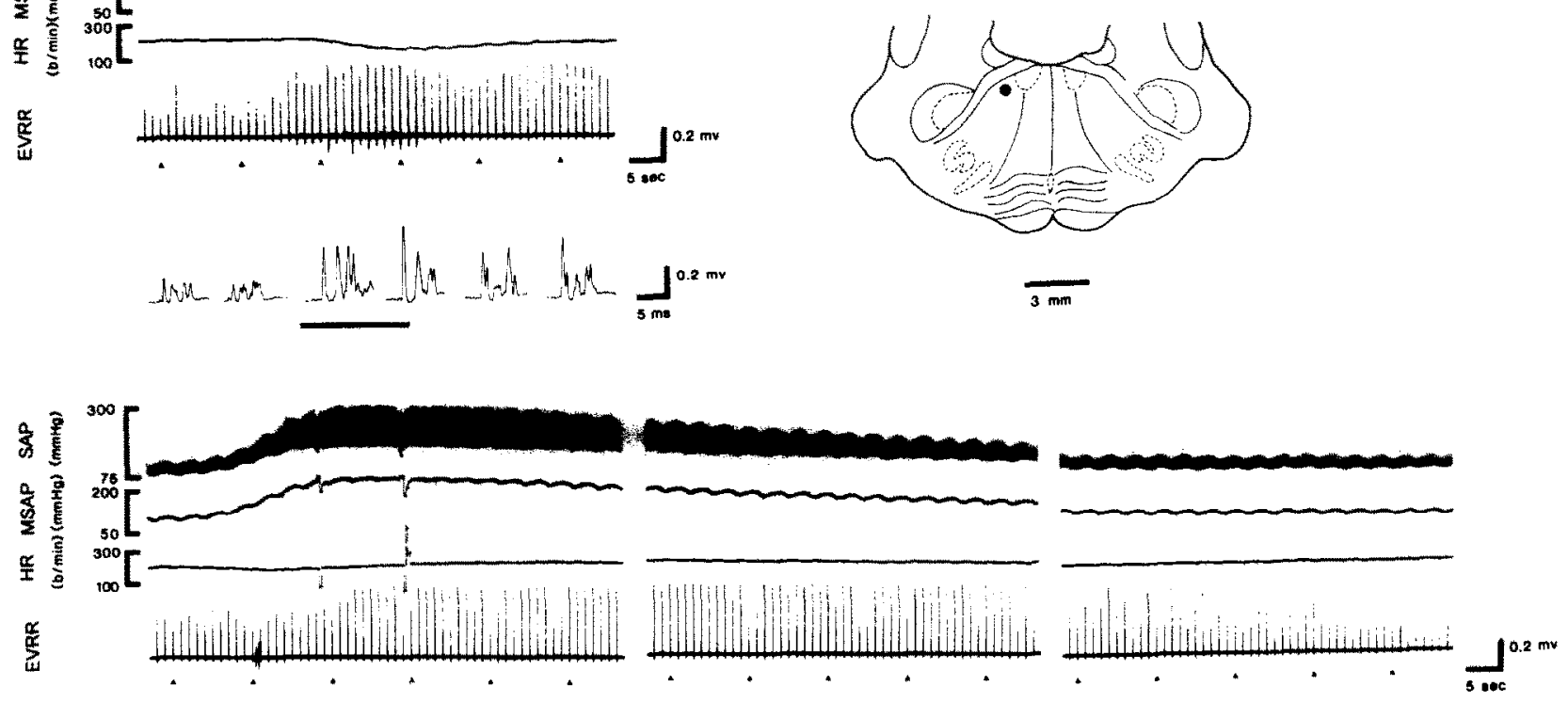

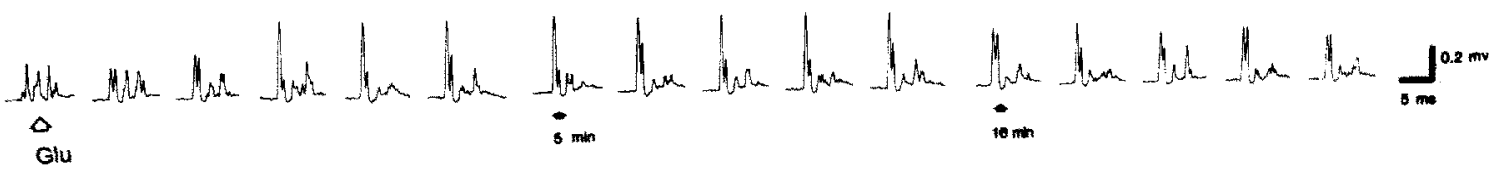
FIG. 4.

398 

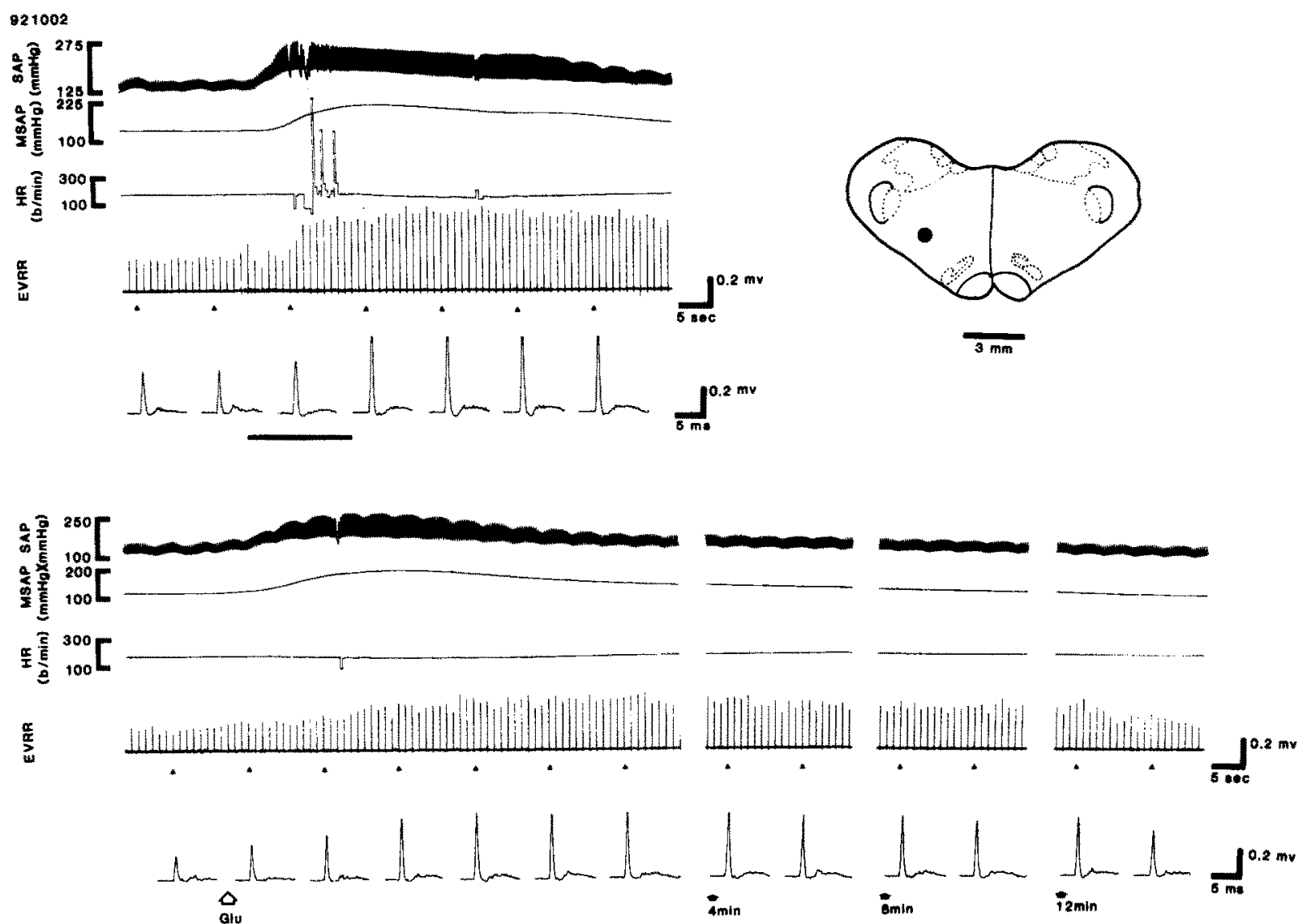

FIG. 5. Stimulation of the ventrolateral medulla (VLM) produced augmentation of the EVRR. Both electrical stimulation (150 $\mu \mathrm{A}$, upper panels) and Glu microinjection (200 nl, lower panels) at a point in VLM, $4 \mathrm{~mm}$ rostral to the obex as shown in the brain drawing, produced increases of SAP and EVRR. Note that both electrical and Glu stimulation produced marked increases of SAP and EVRR. Note also the prolonged action of Glu (12 $\mathrm{min})$.

including medulla. Hypothalamic stimulation as well facilitated the monosynaptic spinal reflex induced by dorsal root stimulation (12). However, these early studies did not examine changes in blood pressure.

In the rostral pons and midbrain pressor areas correlated changes in somatic functions have been observed in the rat, that is, tail-flick reflex $(14,29)$. In cats, PAG stimulation and RP stimulation facilitated the lumbar monosynaptic refiex (31). Similar facilitation of lumbar monosynaptic reflexes was observed on RP stimulation (9). Stimulation of the region ventral to RP, cuneiform nucleus, and the ventral region of the inferior colliculi (20) increased the tonic stretch reflex and biceps muscle tone. Stimulation of the midbrain retrorubal, pedunculopontine tegmental nuclei, and ventral paralemniscal tegmental field suppressed muscle tonus (22).

Consistent with our previous study (8) Glu microinjections into DM and VLM produced both marked increases in SAP and inhibition of EVRR. The inhibition by DM and VLM was about equal. The frequency of a SAP increase and EVRR decrease and an independent EVRR decrease associated without a SAP change was also about equal. Nevertheless, the frequency of producing an independent EVRR increase associated without a SAP change was higher in VLM than DM. When the VLM is lesioned bilaterally, marked reduction in the EVRR increase occurred in response to DIC, or RP stimulation (Table 2). Therefore, the VLM appears to be an active region with somatic function.

The medullary-pontine parts of the brain stem that is involved in cardiovascular activity also modulate both somatic motor and sensory functions. For instance, stimulation of the medullary reticular formation close to the facial nucleus inhibited the spinal nociceptive reflex (13). Lesioning of this area reduced the nociceptive reflex (19). Stimulation of the ventral root L7 and S1 increased the muscle tonus associated with an increase in VLM neural activity (3). Microinjection of carbachol into the dorso-

FIG. 3. Effects of stimulation of the midbrain periaqueductal grey (PAG) on EVRR. The PAG (dot of the brain drawing) was stimulated first with an electrical current $(300 \mu \mathrm{A}, 1.0 \mathrm{msec}, 80 \mathrm{~Hz}$ and $15 \mathrm{~s})$ and then followed by Glu $(200 \mathrm{nl})$. Note that electrical stimulation produced an increase of SAP and EVRR, while Glu stimulation failed to elicit any pressor response but decreased the EVRR.

FIG. 4. Stimulation of the gigantocellular tegmental field (FTG) produced augementation of the EVRR. Both electrical (150 $\mu$, upper panels) and Glu microinjection (200 $\mathrm{nl}$, lower panels) at a point in RP, $10 \mathrm{~mm}$ rostral to the obex as shown in the brain drawing, produced an increase in SAP and EVRR. Note that electrical stimulation produced a slight decrease, while Glu an insignificant change in HR. Note also that EVRR was not increased until the SAP rise had reached the plateau. 

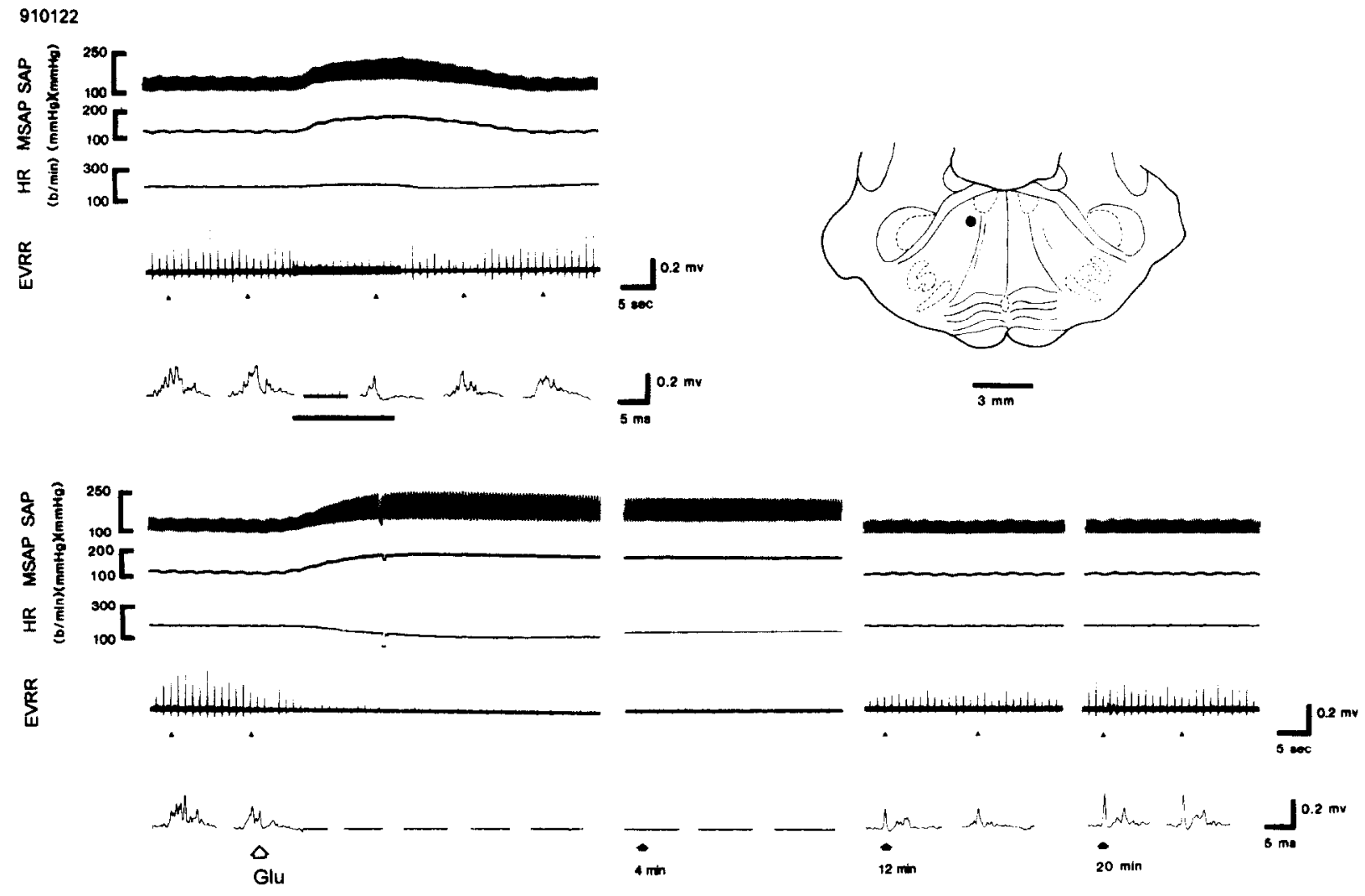

FIG. 6. Stimulation of the gigantocellular tegmental field (FTG) produced inhibition of the EVRR. Upper panels show that electrical stimulation $(150 \mu \mathrm{A})$ of a point in FTG (dot on the brain drawing), $10 \mathrm{~mm}$ rostral to the obex, produced an increase of SAP, slight increase in HR, and a decrease of EVRR. Microinjection of Glu (200 nl) into the same site (lower panels) produced similar but more prolonged SAP rise and bradycardia. EVRR was completely silent for a period of $12 \mathrm{~min}$ during the period of cardiovascular reaction.
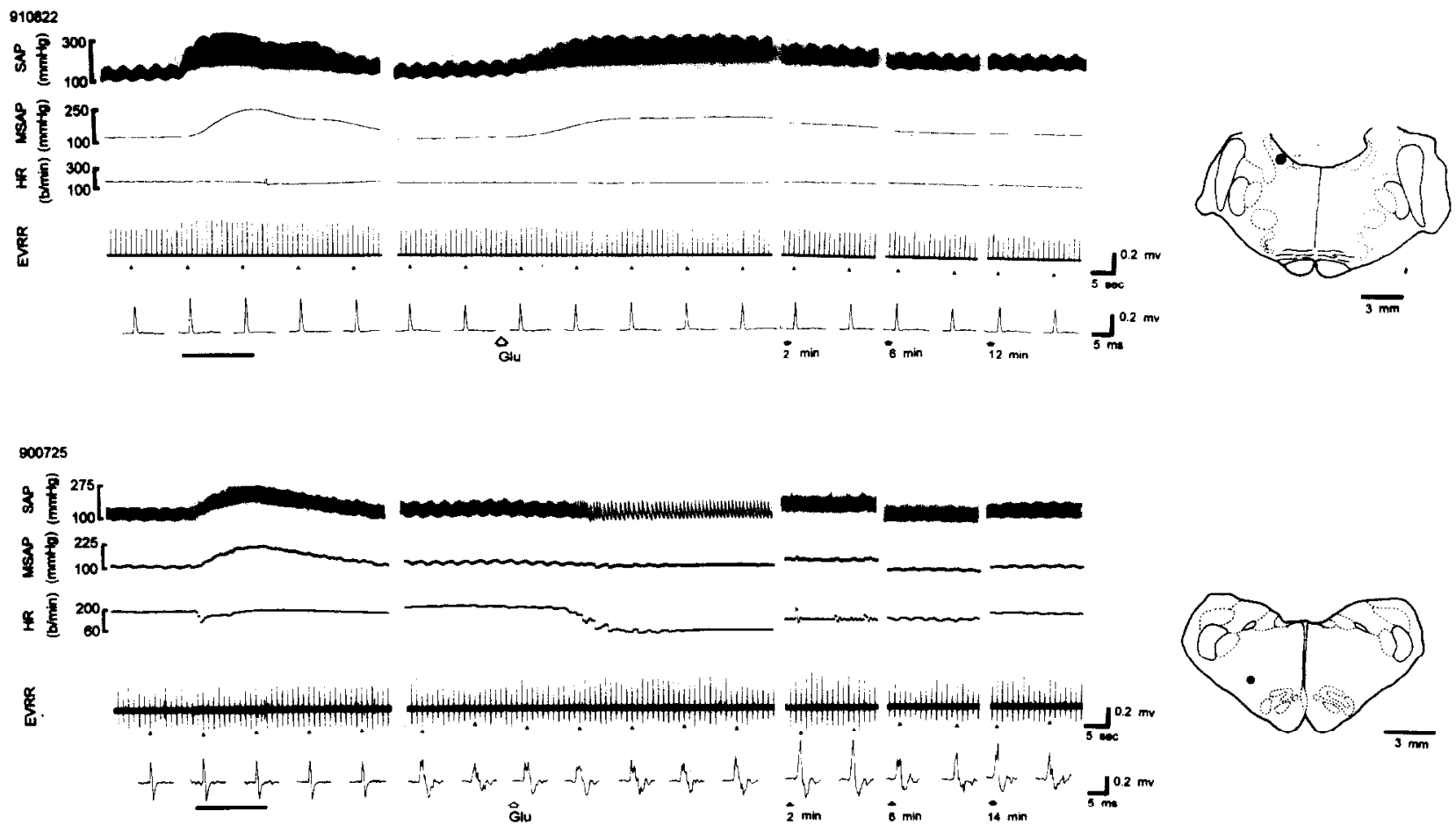

FIG.7. Separation of vasomotor action and inhibition of spinal reflex. Upper panel (cat 910822) show that both electrical stimulation (150 $\mu \mathrm{A})$ and Glu (200 nl) at a point in LC as shown in the brain drawing produced elevation of SAP, but no change in EVRR. Lower panel (cat 900725 ) show that at a point in the VLM (dot in the brain drawing) electrical stimulation $(150 \mu \mathrm{A})$ did not change the EVRR. In this case, electrical stimulation produced a marked pressor response associated with a brief bradycardia. Glu ( $200 \mathrm{nl}$ ), produced a marked bradycardia, but was not associated with a change in SAP. The EVRR did not change during the period of bradycardia but began to increase after the bradycardiac response was over. 
TABLE 2

EFFECTS OF BILATERAL DC LESIONS ON VARIOUS PRESSOR AREAS OF THE BRAIN STEM ON THE AUGMENTED EVRR PRODUCED BY ELECTRICAL STIMULATION OF THE DIENCEPHALON, MIDBRAIN, AND ROSTRAL PONS

\begin{tabular}{llc}
\hline \multicolumn{1}{c}{ Brain Stimulation } & $\begin{array}{c}\text { SAP Increase } \\
(\%)\end{array}$ & $\begin{array}{c}\text { EVRR Increase } \\
(\%)\end{array}$ \\
\hline Diencephalon & & \\
$\quad$ Control & $37.9 \pm 6.7$ & $40.7 \pm 10.0$ \\
After PAG lesion $(n=5)$ & $22.5 \pm 5.8$ & $23.4 \pm 10.4^{*}$ \\
Control & $25.3 \pm 4.5$ & $38.9 \pm 9.3$ \\
After RP lesion $(n=5)$ & $30.3 \pm 11.3$ & $29.9 \pm 10.4$ \\
Control & $38.6 \pm 5.4$ & $76.4 \pm 28.9$ \\
After VLM lesion $(n=7)$ & $14.8 \pm 3.7^{*}$ & $43.7 \pm 9.3^{*}$ \\
Control & $48.7 \pm 10.4$ & $70.9 \pm 16.9$ \\
After DM lesion $(n=7)$ & $12.6 \pm 7.2^{*} \dagger$ & $82.2 \pm 21.3$ \\
Midbrain & & \\
Control & $40.9 \pm 6.5$ & $41.6 \pm 11.4$ \\
After RP lesion $(n=5)$ & $35.9 \pm 5.1$ & $63.7 \pm 11.4^{*}$ \\
Control & $47.6 \pm 8.2$ & $56.0 \pm 15.2$ \\
After VLM lesion $(n=8)$ & $15.4 \pm 5.0^{*} \dagger$ & $105.1 \pm 32.7^{*}$ \\
Control & $59.3 \pm 11.1$ & $46.0 \pm 14.2$ \\
After DM lesion $(n=8)$ & $42.5 \pm 7.8$ & $60.8 \pm 21.5$ \\
Rostral Pons & & \\
Control & $51.8 \pm 9.3$ & $42.9 \pm 10.4$ \\
After VLM lesion $(n=7)$ & $22.2 \pm 8.8^{*} \dagger$ & $24.3 \pm 12.3^{*}$ \\
Control & $73.5 \pm 10.4$ & $55.7 \pm 14.9$ \\
After DM lesion $(n=7)$ & $61.5 \pm 17.0$ & $53.0 \pm 17.0$ \\
\hline
\end{tabular}

Values are mean $(\%) \pm S E$.

* Indicates that in the same animal groups, the percent changes of the EVRR before and after brain lesions are statistically significant ( $p$ $<0.05$ ), verified by paired Student's $t$-test.

I Indicates that in the pooled animal group, the percent changes of the EVRR before and after brain lesions are statistically significant $(p$ $<0.05$ ), verified by one-way ANOVA and compared by pairs.

lateral pontine reticular formation suppressed muscle tone (EMG) (21) and the ventral root (L7) evoked response following sciatic nerve stimulation (26). Also carbachol microinjection into the nucleus reticularis gigantocellularis inhibited the evoked ventral root discharges in response to tibial nerve stimulation (27).
Both the DM and VLM are involved in the modulation of sympathetic reflex activity. For example, stimulation of the sciatic, hamstring, or superficial radial nerve produced an carly inhibition (silent) followed by a late excitation of the evoked potential recorded at the renal branch of the splanchnic nerve. The inhibitory component was abolished by bilateral VLM lesions while the late excitatory component was abolished by bilateral DM lesions (16). Lesions of the VLM abolished the vasopressor response resulting from stimulating sciatic afferents $(10,30)$. Cooling of the VLM produced a marked reduction of the somato-sympathetic reflex, a spinal component of the potential recorded at the $\mathrm{T} 3$ sympathetic white ramus following stimulation of the correspondent intercostal nerve (11). Lesions of VLM abolished the increase in SAP and heart rate following stimulation of the lumbar and sacral ventral toots (17). VLM neurons also respond to stimulation of the radial or sciatic nerves (28). Furthermore, more than half of the responses in VLM, which were correlated with the cardiac rhythm, increased firing during muscle contraction response to ventral root $(\mathrm{L} 7$, S1) stimulation (2).

These results do not explain the mechanism of interaction between EVRR and changes in SAP following brain stem stimulation. The modulation of cardiovascular function and somatic reflex activity are most likely mediated by different neurons in close proximity in one another with the possibility and advantage of interaction at various levels in the brain stem and spinal card. Consequently, simultaneous mobilization of both systems is possible in an emergency.

In summary, we describe the activity of two types of neurons which mediate cardiovascular or somatic effects in the diencephalon, rostral pons, midbrain, particularly the periaqueductal grey, dorsal medulla and ventral lateral medulla. These neurons with cardiovascular and somatic properties are distinct populations in close proximity with a closer association in the medullary regions than in the structures within or rostral to the midbrain.

\section{ACKNOWLEDGEMENTS}

The authors thank Mr. G. T. Chen for preparation of the illustrations and Ms. J. J. Pan and Y. M. Wu for preparation of the manuscript. This study was supported in part by the Foundation of Biomedical Sciences, Institute of Biomedical Sciences, Academia Sinica, Shih-chun Research Fund and the National Science Council, R.O.C. No. NSC80-0412B001-02.

\section{REFERENCES}

1. Blander, R.; Carrive, P.; Zhang, S. P. Integration of somatic and autonomic reactions within the midbrain periaqueductal grey: Viscerotopic, somatotopic and functional organization. Progress in Brain Res. 87:269-305; 1991.

2. Bauer, R. M.; Iwamoto, G. A.; Waldrop, T. G. Discharge patterns of ventrolateral medullary neurons during muscular contraction. Am. J. Physiol. 259:R606-R611; 1990.

3. Bauer, R. M.; Waldrop, T. G.; Iwamoto, G. A.; Holzwarth, M. A. Properties of ventrolateral medullary neurons that respond to muscular contraction. Brain Res. Bull. 28:167-178; 1992.

4. Chai, C. Y.; Lee, T. M.; Wayner, M. J. Effect of visceral afferent activation on leg extension induced by sciatic afferent stimulation. Brain Res. Bull. 3:115-123; 1977.

5. Chai, C. Y.; Lin, Y. F.; Lin, A. M. Y.; Pan, C. M; Lee, E. H. Y.; Kuo, J.S. Existence of a powerful inhibitory mechanism in the medial region of caudal medulla - with special reference to the paramedian reticular nucleus. Brain Res. Bull. 20:515-528; 1988.

6. Chai, C. Y.; Lin, R. H.; Lin, A. M. Y.; Pan, C. M.; Lee, E. H. Y.; Kuo, J. S. Pressor responses from electrical or glutamate stimula- tions of the dorsal or ventrolateral medulla. Am. J. Physiol. 255:R709-R717; 1988

7. Chai, C. Y.; Lin, Y. F.; Wang, H. Y.; Wu, W. C.; Yen, C. T.; Kuo, J. S.; Wayner, M. J. Inhibition of spinal reflexes by paramedian reticular nucleus. Brain Res. Bull. 25:581-588; 1990.

8. Chai, C. Y.; Wu, W. C.; Wang, S.; Su, C. K.; Lin, Y. F.; Yen, C. T.; Kuo, J. S.; Wayner, M. J. Coexistence of autonumic and somatic mechanisms in the pressor areas of medulla in cats. Brain Res. Bull. 29:15-26; 1992.

9. Chan, J. Y. H.; Fung, S. J.; Chan, S. H. H.; Barnes, C. D. Facilitation of lumbar monosynaptic reflexes by locus coeruleus in the rat. Brain Res. 369:103-109; 1986.

10. Ciriello, J.; Calaresu, F. R. Lateral reticular nucleus: A site of somatic and cardiovascular integration in the cat. Am. J. Physiol. 233:R100-R109; 1977.

11. Dembowsky, K.; Lackner, K, Czachurski, J.; Seller, H. Tonic catecholaminergic inhibition of the spinal somato-sympathetic reflexes originating in the ventrolateral medulla oblongata. J. Auton. Nerv. Sys. 3:277-290; 1981 
12. Feldman, S; Wagman, I. H. Hypothalamic effects on spinal reflexes and their alteration by pentobarbital. Exp. Neurol. $5: 250-268 ; 1962$.

13. Gebhart, G. F; Sandkuhler, J.; Thalhammer, J. G.; Zimmermann, M. Inhibition of spinal nociceptive information by stimulation in midbrain of the cat is blocked by lidocaine microinjected in nucleus raphe magnus and medullary reticular formation. J. Neuroci. 50:1446-1459; 1983.

14. Gebhart, G. F.; Ossipov, M. H. Characterization of inhibition of the spinal nociceptive tail-flick reflex in the rat from the medullary lateral reticular nucleus. J. Neurosci. 6:701-713;1986.

15. Goodchild, A. K.; Dampney, R. A. L.; Bandler, R. A. Method for evoking physiological responses by stimulation of cell bodies but not axons of passage within localized regions of the central nervous system. J. Neurosci. Meth. 6:351-363; 1982.

16. Iwamura, Y.; Uchino, Y.; Ozawa, S.; Kudo, N. Excitatory and inhibitory components of somato-sympathetic reflex. Brain Res. 16:351 358; 1969.

17. Iwamoto, G. A.; Kaufman, M. P.; Botterman, B. R.; Mitchell, J. H. Effects of lateral reticular nucleus lesions on the exercise pressor refiex in cats. Circ. Res. 51:400-403; 1982.

18. Janss, A. J.; Cox, B. F.; Brody, M. J.; Gebhart, G. F. Dissociation of antinociceptive from cardiovascular effects of stimulation in the lateral reticular nucleus in the rat. Brain Res. 405:140-149; 1987.

19. Janss, A. J.; Gebhart, G. F. Brainstem and spinal pathways mediating descending inhibition from the medullary lateral reticular nucleus in the rat. Brain Res. 440:109-122; 1988.

20. Juch, P. J. W.; Schaafsma, A.; Van Willigen, J. D. Brainstem influences on biceps reflex activity and muscle tone in the anaesthetized rat. Neurosci. Lett. 140:37-41; 1992.

21. Lai, Y. Y.; Siegel, J. M. Cardiovascular and muscle tone changes produced by microinjection of cholinergic and glutamatergic agonists in dorsolateral pons and medial medulla. Brain Res. 514:27$36 ; 1990$.

22. Lai, Y. Y.; Siegel, J. M. Muscle tone suppression and stepping pro- duced by stimulation of midbrain and rostral pontine reticular formation. J. Neurosci. 10:2727-2734; 1990.

23. Lin, A. M. Y.; Wang, Y.; Kuo, J. S.; Chai, C. Y. Homocysteic acid elicits pressor responses from ventrolateral medulla and dorsomedial medulla. Brain Res. Bull. 22:627-631; 1989.

24. Lin, R. H.; Lin, A. M. Y.; Su, C. K.; Kuo, J. S.; Chai, C. Y. Presence of perikarya for vasopressor actions in both the dorsal and ventrolateral regions of medulla oblongata in swine. Neurosei. Res. Comm. 5:125-133; 1989.

25. Magoun, H. W; Rhines, R. An inhibitory mechanism in the bulbar reticular formation. J. Neurophysiol. 9:165-171; 1946.

26. Morales, F. R; Engelhardt, J. K.; Soja, P. J.; Pereda, A. E; Chase M. H. Motoneuron properties during motor inhibition produced by microinjection of carbachol into the pontine reticular formation of the decerebrate cat. J. Neurophysiol. 57:1118-1129; 1987.

27. Pereda, A. E.; Morales, F. R.; Chase, M. H. Medullary control of lumbar motoneurons during carbachol-induced motor inhibition. Brain Res. 514:175-179; 1990.

28. Rosen, 1.; Scheid, P. Patterns of afferent input to the lateral reticular nucleus of the cat. Exp. Brain Res. 18:242-255; 1973.

29. Sandkuhler, J.; Gebhart, G. F. Characterization of inhibition of a spinal nociceptive reflex by stimulation medially and laterally in the midbrain and medulla in the pentobarbital-anesthetized rat. Brain Res. 305:67-76; 1984.

30. Stornctta, R. L.; Morrison, S. F; Ruggiero, D. A.; Reis, D. J. Neurons of rostral ventrolateral medulla mediate somatic pressor reflex. Am. J. Physiol. 256:R448-R462; 1989.

31. Strahlendorf, J. C.; Strahlendorf, H. K.; Kingsley, R. E. ; Gintautas, J.; Barnes, C. D. Facilitation of the lumbar monosynaptic reflexes by locus coeruleus stimulation. Neurophamacol. 19:225-230; 1980

32. Su, C. K.; Yen, C. T.; Hwang, J. C.; Tseng, C. J.; Kuo, J. S.; Chai, C. Y. Differential control of sympathetic nerve activities by the pressor mechanisms of dorsal and rostral ventrolateral medulla oblongata in cats. J. Auton. Nerv. Syst, 40:141-154; 1992. 